\title{
Astronomy in post-apartheid South Africa
}

\author{
Patricia Ann Whitelock ${ }^{1,2}$ \\ ${ }^{1}$ South African Astronomical Observatory, \\ PO Box 9, Observatory 7935, South Africa \\ email: paw@saao.ac.za \\ ${ }^{2}$ National Astrophysics and Space Science Programme, \\ Department of Astronomy, University of Cape Town, South Africa
}

\begin{abstract}
Astronomy was one of the sciences earmarked for major support by South Africa's first democratically elected government in 1994. This was a very remarkable decision for a country with serious challenges in poverty, health and unemployment, but shows something of the long term vision of the new government. In this paper I give one astronomer's perception of the reasons behind the decision and some of its consequences.
\end{abstract}

Keywords. Africa, education, development, SALT, SKA

\section{Introduction}

To set the scene I start with a brief description of the astronomical environment as it is in South Africa (SA) right now (early 2009), before stepping back to look at the strategic thinking that brought us here. Within this context I highlight some of the most significant political documents and legislation. I end with a brief look at the size and distribution of the astronomy community and say something of our efforts to grow and nurture the next generation of users for our facilities.

While I do my best to be objective, this is a personal account from the viewpoint of an optical astronomer living in Cape Town and working primarily for the South African Astronomical Observatory. Others would inevitably see things differently and place the emphasis elsewhere.

\section{Astronomy research facilities}

The existing astronomical facilities, and the SA contribution to international facilities are financed by government through the Department of Science and Technology (DST). The two major observatories mentioned below: The South African Astronomical Observatory (SAAO) and the Hartebeesthoek Radio Observatory (HartRAO) are administered as National Research Facilities within the National Research Foundation (NRF). This arrangement could change after the formation of a South African Space Agency in 2009, but that remains to be seen.

\subsection{Optical Astronomy}

The SAAO is the national observatory for optical astronomy. Its headquarters are in Cape Town while its telescopes are $360 \mathrm{~km}$ by road to the north-east near the small town of Sutherland, in the Northern Cape. The small telescopes owned by SA predate the current era, the largest of them, a $1.9 \mathrm{~m}$, being over 50 years old. These are still used by local and international astronomers. They have been well maintained until very recently and have the potential to go on being useful research tools provided their instrumentation 


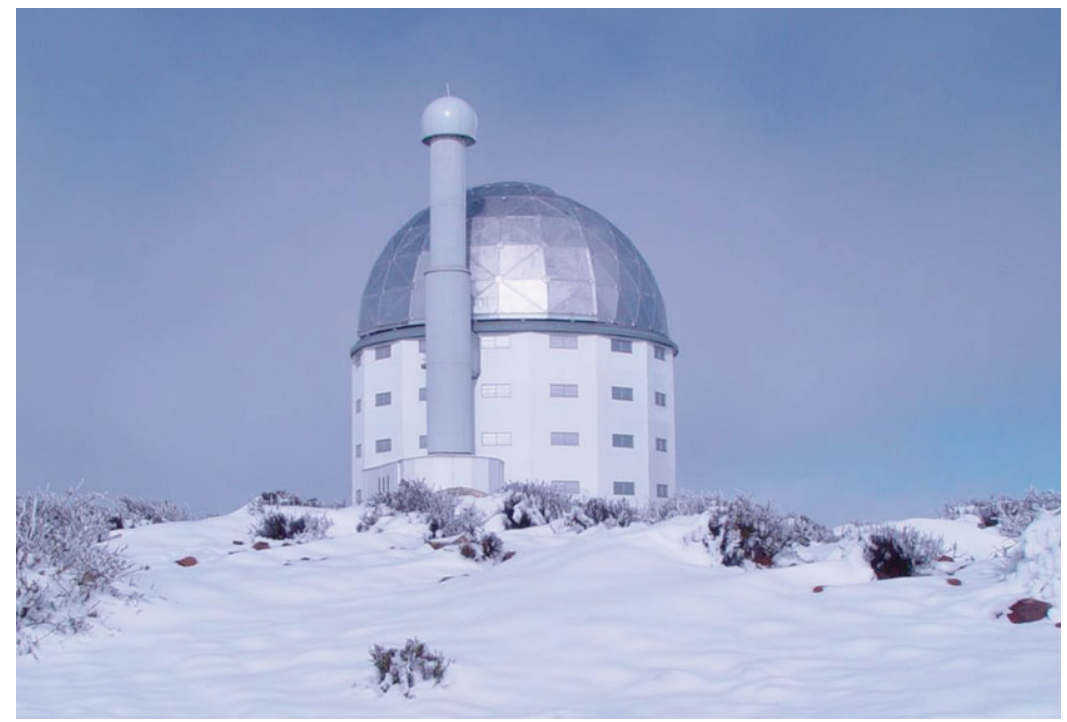

Figure 1. SALT in the African winter (credit: SAAO)

can be renewed on a regular basis. At present SAAO is concentrating its limited resources on the new large telescope, SALT.

There are also newer small telescopes that have been located at Sutherland because of its climate, position and infrastructure. Many of these are run as joint projects between $\mathrm{SA}$ and an international partner - the $1.4 \mathrm{~m}$ Japanese-South African Infrared Survey Facility (IRSF) is the largest of these and has been very successfully used for research and training since its opening in 2000. There are a number of small automated telescopes run for specific projects on behalf of individual institutes or consortia, including groups from Korea, UK, USA and Germany. There are also a variety of non-astronomical projects, such as a seismograph and a geodynamic observatory, located at Sutherland for reasons of geography, geology (high seismic stability) and infrastructure.

\subsubsection{The Southern African Large Telescope (SALT)}

SALT (see Fig. 1) is a 10m-class telescope with a segmented spherical primary mirror, similar, but not identical, to the Hobby-Eberly Telescope in Texas, USA. SALT is owned by an international consortium within which SA is the largest single shareholder. The shareholders include consortia and universities from USA, Poland, India, Germany, UK and New Zealand (www.salt.ac.za). It has been scientifically productive since 2006 (see O'Donoghue et al. 2006) although some residual problems with the image quality are only now being dealt with. Although capable of working up to almost $2 \mu \mathrm{m}$, SALT has been optimized for use in the near-ultraviolet, up to the atmospheric cut-off, and will therefore be very complementary to various large infrared telescopes now proliferating.

\subsection{Radio Astronomy}

The Hartebeesthoek Radio Observatory (HartRAO) is the national facility for radio astronomy and has operated a 26m radio telescope since 1974 (www.hartrao.ac.za and Fig. 2). The antenna was built by NASA in 1961, originally as part of their deep space network for tracking spacecraft beyond Earth orbit. Because of its unique location on the southern plate it has played an important role in the world's VLBI networks for both radio astronomy and geodesy. In the period 2000 to 2004 the surface of the antenna 


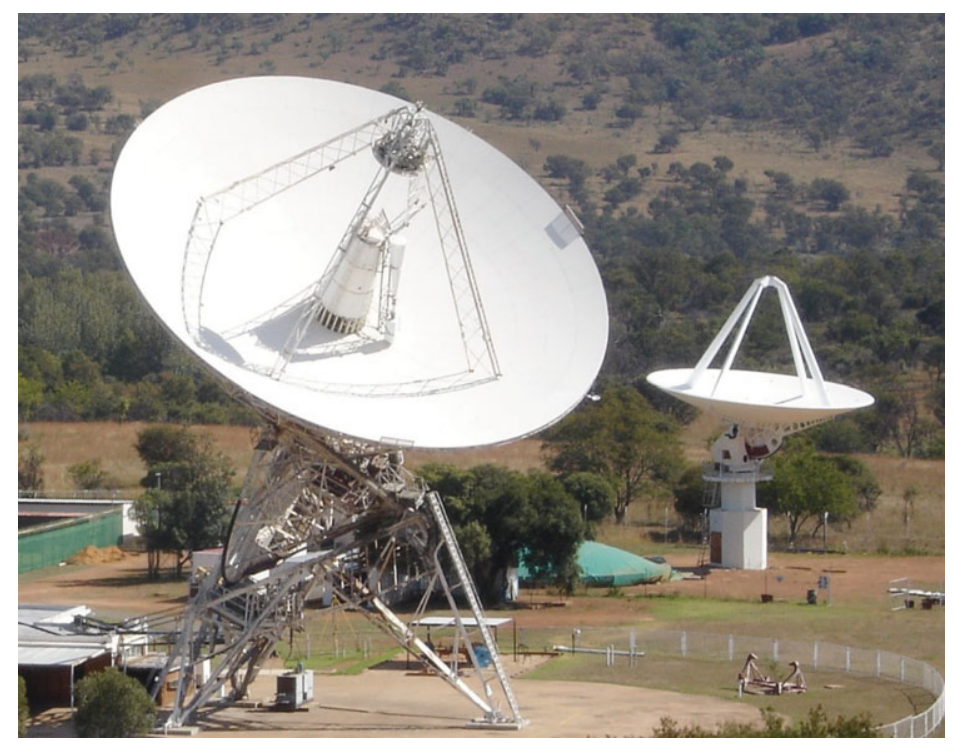

Figure 2. HartRAO with the $26 \mathrm{~m}$ dish and the $15 \mathrm{~m}$ eXperimental Development Model (XDM) test-bed prototype for KAT (credit: Mike Gaylard)

was upgraded to enable it to operate at frequencies above $15 \mathrm{GHz}$. Unfortunately, in late 2008 the antenna suffered a failure of the major bearing on the polar drive shaft. Given its international importance, the observatory, supported by the NRF, is currently considering two options: a repair, which will be costly and not without risk, and the purchase of a new antenna.

During 2007 a 15m antenna of innovative design (composite one piece reflector) was constructed at Hartebeesthoek as a test-bed prototype for the Karoo Array Telescope (see below and Fig. 2).

\subsection{Square Kilometre Array (SKA) Karoo Array Telescope (KAT)}

South Africa is on the short list, together with Australia, to host the Square Kilometer Array (SKA) radio telescope. The Karoo Array Telescope will be used to test technology leading up to the development of the SKA and will be a very powerful telescope in its own right (www.ska.ac.za). The first seven antennae for KAT are under construction in the Karoo and should be operational later this year. The full array, of at least 80 dishes, is scheduled for 2012 and will be known as MeerKAT (meer is Afrikaans for more). If the SKA itself goes to SA the central compact array will be located near Carnarvon, which is an area with particularly low radio interference and will, in future, be protected by legislation (see below). The exact disposition of the other dishes has yet to be settled, but spreading them throughout Southern Africa, as far east as Mauritius and as far north-west as Ghana, is quite possible.

\subsection{Other Wavelengths}

SA is also a partner in the highly successful international collaboration which operates the High Energy Stereoscopic System (HESS) in Namibia. We anticipate participating in HESS-II, currently under construction and other high energy experiments still under discussion. While SA is not a big player in these activities our participation gives us access to important wavelength regimes as well as offering partnerships that involve our neighbouring countries. 


\section{Political milestones}

In 1994 SA held its first democratic elections and Nelson Mandela became President. His government created a Department of Arts, Culture, Science and Technology (DACST) $\dagger$. In 1996 DACST produced a White Paper on science and technology which prioritized:

- improved quality of life for all

- a competitive economy

- a democratic culture

within a National System of Innovation (NSI) that made capacity development a priority.

Interestingly from our perspective this White Paper also discussed the role of pure science, and included the following statement:

"Scientific endeavour is not purely utilitarian in its objectives and has important associated cultural and social values. It is important to maintain a basic science competence in 'flagship' sciences such as physics and astronomy for cultural reasons. Not to offer them would be to take a negative view of our future - the view that we are a second-class nation, chained forever to the treadmill of feeding and clothing ourselves."

The idea that astronomy is particularly important to us continued to be developed and I want to mention here two items from the DST strategy document released in 2002. First, the specific statement that:

Clearly, with limited resources our best chances of success will depend on our ability to focus on our potential strengths while staying well connected to international research.

Secondly, the document lists areas where South Africa has an obvious geographical advantage as follows:

(a) Astronomy

(b) Human paleontology

(c) Biodiversity

(d) Antarctic research

Other areas, such as indigenous knowledge and the technology for deep mining were highlighted as areas in which the country had a special knowledge advantage. It is interesting to see astronomy at the top of this list and much of what has happened since should be seen in the light of this desire to exploit our geographical advantage by international collaboration, but with a strong development objective in view.

In 2007 some important legislation was passed by parliament to recognize and protect SA's strategic advantage in astronomy. The Astronomy Geographic Advantages (AGA) Bill aims to protect sites such as Sutherland and the area around the Karoo Array Telescope from electromagnetic pollution, dust etc. The intension is that SA will be able to participate in global scale science projects as a partner in which this country provides the location and an enabling environment, while international partners provide technical and financial resources. We are currently in the process of defining the detailed regulations that go with the legislation and I think the optical astronomers have a much easier road here than our radio colleagues. It will be interesting to see where the political will is if the astronomers clash with the miners.

$\dagger$ This is a combination of activities that the participants of this conference might be particularly pleased to see, as was I at the time. So you may be saddened to hear that it did not work and in 2002 a separate department was created for Science and Technology. 
In the 2008 ten year innovation plan DST take a slightly different approach from the earlier strategic plan. This highlights five "grand challenge areas" as follows:

- The Farmer to Pharma value chain to strengthen the bio-economy

- Space science and technology

- Energy security

- Global-change science with a focus on climate change

- Human and social dynamics

Astronomy is located here as one of the space science; indeed three of the eight illustrations within this document are astronomical. At the time of writing legislation has been passed that enables the formation of a Space Agency. That Agency will come into being later in 2009, but the exact relationship of the astronomical facilities to it has yet to be defined.

\section{Decision to build SALT}

The SA astronomy community had been talking about the need for a new, larger telescope since the 1980s; pointing out that the $1.9 \mathrm{~m}$ telescope (originally in Pretoria, now at Sutherland) when constructed in the 1950s was the largest telescope in the Southern hemisphere. It had since been overtaken many times; it was obsolete and the community was no longer competitive. This view had received considerable support from science organizations within the country and international panels. Various ideas around a new $4 \mathrm{~m}$ were developed and discussed, but it was only in 1996 when the idea of a copy of the Hobby-Eberly telescope (HET) was introduced that the political interest become real. The HET was nearing completion in Texas and a copy of this would be the largest single telescope in the Southern Hemisphere, not just another big telescope. At US $\$ 20$ million the estimated price of construction was affordable and the idea that you got $70 \%$ of the sky for $20 \%$ of the price, seemed 'cost effective' and appealed to all. The case for SALT was put together by a combination of people from DST, FRD (the forerunner of NRF) and SAAO and the document that went to Cabinet emphasized the following:

- Strength of SA astronomy and potential for new international collaborations

- Location - bringing together the geographic advantage (latitude, longitude, climate, geological stability etc.), the low labour costs and perhaps most importantly the fact that Sutherland was in the Northern Cape Province - this is a poorly resourced underdeveloped part of the country with almost no science/technology going on and a lot of political pressure to make something happen.

- Focus for competence. The skills involved in building, then operating, a telescope are broadly useful ones that are required in other areas.

- Science Education

- Science Awareness - the arguments about education and public awareness are of course the same in Africa as they are worldwide

- 'African Flagship'. This one should not be underestimated we need things that show each other and the world that Africa can do high-tech science successfully

- Benefits to SA industry. These are best illustrated by the fact that SA put in one third of the cost of building SALT, but two thirds of the cost were spent within the country. This is clearly the same sort of requirement that more developed nations have when it comes to big projects

On 1 June 1998 the Minister of Arts, Culture, Science and Technology announced during his budget speech in parliament that SA would fund $50 \% \dagger$ of the construction of

$\dagger$ SA's final share was nearer $30 \%$, mostly due to changes in the Rand/Dollar exchange rate. 
SALT. The decision had been taken by Cabinet and the commitment was for 50 million Rand (about US $\$ 10$ million at the time). The remaining $50 \%$ had to be raised through international partnerships. In 2000 sufficient funds were available and construction was started with a ground breaking event in which the Minister of Science and Technology and the Premier of the Northern Cape played prominent roles.

SALT has been set up as a private "not for profit" company, called the SALT Foundation, in which all the partners are shareholders. Its purpose is to advance science and training through the promotion of astronomy and astrophysics in the Southern hemisphere and to enhance collaboration between scientists and countries in these fields.

\section{SALT as a tool for development}

SALT was constructed by a largely SA team hired by the SALT Foundation, and is operated by the SAAO under contract to the Foundation. The primary focus of that Foundation is on the functioning of the telescope and on its astronomical output. For SA, with its challenges of basic education and poverty, this could never be enough and various people, most prominent among them the then President of the FRD/NRF, ensured that there was a vision that went beyond science.

What they started is known as the SALT Collateral Benefits Plan, the primary idea being that within SA SALT must do more than science if it is to be a success. To quote the plan itself: "SALT must provide distinct and substantial benefits in the development of people, technology and the economy, through:

- Educational empowerment (tertiary sector)

- Public outreach

- Direct educational benefits (educators and learners)

- Science education visitor centres

- SALT as an African Facility

SALT must make a major impact on society via the opportunities it presents for a new generation of South Africans to transcend the inequalities of the past and leap over the problems of today."

All of this can only be meaningful if SALT actually functions as a first rate telescope, and it is not there yet. Some more details of recent SALT education and outreach activities are given by Govender (2009) who is currently in charge of the SALT collateral benefits division of SAAO.

SALT was formally opened in 2005 by then President Thabo Mbeki. His speech made it very clear that a great deal was expected from the telescope and its users, in particular:

"Out of this place, enveloped by the quiet peace of the Karoo and its starlit skies, must and will come the message that thought is humanity's stepladder out of Hades that ignorance is nothing but condemnation to live for eternity in the world inhabited by the souls of the dead."

\section{The Astronomy Community}

The community of professional astronomers has doubled in the last 15 years, but still remains small. There are about $60 \mathrm{PhD}$ astronomers in the country, almost 40 of them based in Cape Town (at SAAO, University of Cape Town, University of the Western Cape and the KAT office). The largest number of people are engaged in optical astronomy; the radio community is tiny, but growing rapidly. There are rather few theorists although there is a strength in theoretical cosmology. It is particularly encouraging to see the University of Cape Town (UCT) establishing a centre of excellence in astronomy and 
cosmology, which will facilitate the interaction of theoreticians and observers within that university, which, other than SAAO, is by far the largest grouping of astronomers.

Nevertheless, the size of the community is not commensurate with the facilities at their disposal and we are in danger of running excellent facilities simply for the benefit of our international partners. Unless the situation is rectified the resulting impact of astronomy as a tool for development will fall very short of what it might be.

\subsection{The National Astrophysics and Space Science Programme}

In 2000, during strategic planning exercises which followed the South African commitment to build SALT, the lack of SA astronomers, and the extreme lack of black astronomers was identified as the biggest threat to the future of astronomy in the country. At the same time we recognized that the lack of high level skills in science and technology was a serious national problem. Realizing that a major part of the problem was the fact that very few students went on to postgraduate studies, we started a national programme that would take physics graduates and prepare them to go on to do PhDs. This was set up in a unique collaboration involving 9 universities and 3 national facilities, based at UCT. The students come from all over SA and many from elsewhere in Africa. The provision of sensible bursaries was a vital part of making this work and initially that was made possible by the Ford Foundation and later the Mellon Foundation. In recent time most of the finance has come from the SA government. Ford still provide PhD bursaries for NASSP graduates to allow them to complete their studies. NASSP puts a strong emphasis on skills so that students who do not stay in astronomy will take away something useful. The overall aim is to create an African network of astronomers bonded by the common experience of schooling and interlinked both professionally and personally.

The way universities are administered in SA makes these collaborations moderately difficult. Finance depends so heavily on how many students you have that students are often strongly discouraged from moving from one institute to another. This is detrimental to the whole of science, not just to astronomy, as students are commonly "shielded" from information relating to numerous opportunities around the country and abroad. The net effect is that rather few people go on to postgraduate studies. One of the aims of the NRF PhD programme (www.nrf.ac.za) is to address these challenges.

Given the prevailing climate it is encouraging that NASSP has not only held together, but grown over the last 6 year. The institutions which participate in NASSP are listed on the web page www.star.ac.za. Starting last year, thanks to the Kellogg foundation and the US National Society of Black Physicists, we also have African American students and faculty participating in NASSP. In 2008 I was approached by a group of environmental scientists who had been told by the Department of Science and Technology to talk to the astronomers and to put something together that was similar to the National Astrophysics and Space Science Programme. That NASSP is seen as a potential role model is also encouraging.

When we first started thinking about NASSP I used the links on the AAS web page to contact a number of American astronomers who had PhDs in astronomy, but who worked outside the field, and asked them what they thought their education gave them that employers found useful. From these exchanges, which were immensely useful, I put together this list:

- Mathematical and statistical abilities

- Problem solving, particularly quantitative solutions

- Data analysis, particularly large quantities of data

- Computer programming, particularly modeling 
- Science communications, verbal and written

- Ability to learn without being taught

These are the skills we try to provide the NASSP students with and I note that there is a good deal of overlap in this list and that given by Jones (2009) in her presentation at this meeting (see page 564).

NASSP is constructed as a one year honours programme followed by an 18 month MSc programme involving 6 months course work then 12 months mini-thesis. We have between 15 and 20 people a year going through the honours and the masters programmes - not huge numbers, but better than the one or two per year before NASSP started. The recruitment from Africa has worked well and we have had students from Botswana, Ethiopia, Gabon, Kenya, Madagascar, Mozambique, Rwanda, Sudan, Uganda, Zambia and Zimbabwe. The African students are quite well educated in theoretical physics, but commonly lack the practical and computer skills you would expect in Europe or North America.

Where we are still falling down is with SA black students. In general we have only one or two per year, so we have put in place a special programme to improve the throughput. This takes disadvantaged students and puts them through an extra year where they get an introduction to astronomy, but mostly they learn the physics and mathematics they will need to succeed with NASSP honours.

We are reasonably pleased at our success in attracting women into NASSP: $27 \%$ of the graduates are women and several of the top students have been women. $27 \%$ is not perfect, but it is very much better than in other physical science courses in SA.

The fundamental problem is still with the school system. A recent OECD (2007) survey of SA specifically mentioned the problems of maths and science teaching. Black people were not allowed to study science to any significant level during apartheid and the democratic government has failed to put into place effective policies to rectify that legacy. The fact, that in 2004 only 2406 black Africans ( $0.5 \%$ of the total) matriculated in mathematics at grade $\mathrm{C}$ or above, illustrates the problem. Of course to go to university and study physics and astronomy you need science as well as mathematics, so the numbers qualifying are even smaller. Everyone is competing for the small number of students and courses like accountancy and engineering are seen as leading to more lucrative careers. This is a problem that astronomy can make an impact on, but it requires a political solution.

\section{Conclusion}

SA is using astronomy as part of its very broad development strategy. The success of this will depend on many factors, but most importantly on the local political will to deal with the education challenges and the level of engagement by the international astronomy community.

\section{References}

O'Donoghue, D., et al. 2006, MNRAS, 372, 151

OECD 2007, OECD Reviews of Innovation Policy: South Africa 2007, OECD Publishing 